\title{
Rate Control and Quality of Life in Patients With Permanent Atrial Fibrillation The Quality Of Life and Atrial Fibrillation (QOLAF) Study
}

\author{
Takayuki Tsuneda, MD; Takeshi Yamashita, MD*; Masatake Fukunami, MD**; \\ Koichiro Kumagai, MD`; Shin-ichi Niwano, MD \\ Ken Okumura, MD*; Hiroshi Inoue, MD
}

\begin{abstract}
Background The present study aimed to determine whether quality of life (QOL) in permanent atrial fibrillation (AF) patients would be improved by monotherapy with $\beta$-blocker (BB) or calcium antagonist (CAA) as compared with digitalis.

Methods and Results Twenty-nine patients with permanent AF under digitalis were randomized into BB (bisoprolol, atenolol or metoprolol) or CAA (verapamil) monotherapy treatment group. Twenty-five were men and the mean age was $67 \pm 8$ years. After the assigned monotherapy, 12 patients received the other monotherapy in a cross-over fashion. Under each treatment, efficacy of rate control was determined by Holter electrocardiogram (ECG), treadmill testing and QOL questionnaire (Short Form-36 (SF-36) and Quality of Life of Atrial Fibrillation (AFQLQ)), and compared with the baseline digitalis treatment. CAA significantly increased mean and minimum heart rate (HR) in Holter ECG as compared with digitalis, whereas BB increased only minimum HR. Exercise duration in treadmill testing was significantly prolonged by CAA treatment, although it only tended to be prolonged by BB treatment. CAA but not BB improved role function-physical score of SF-36, and frequency and severity of symptoms of AFQLQ.

Conclusion These results indicate that CAA is preferable to digitalis when monotherapy is selected for shortterm improvement of QOL and exercise tolerance in patients with permanent AF. (Circ J 2006; 70: 965-970)
\end{abstract}

Key Words: Beta-blocker; Calcium antagonist; Permanent atrial fibrillation; Quality of life; Rate control

$A$ trial fibrillation $(\mathrm{AF})$ is one of the most common arrhythmias in aged people. It begins as paroxysmal $\mathrm{AF}$ and eventually develops the chronic form by $5.5 \%$ of person-years under conventional antiarrhythmic therapy! The treatment strategy for AF consists of rhythm or rate control. Previous clinical studies clearly indicate that rate control is not inferior to rhythm control in terms of

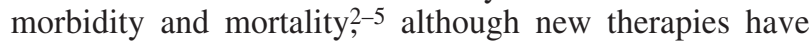
been recently developed for rhythm control (ie, pulmonary vein isolation ${ }^{6}$ or bepridil ${ }^{7,8}$ ). Moreover, a subanalysis of the AFFIRM study showed there were no significant differences in quality of life (QOL) between the rate and rhythm control groups? For patients with symptomatic paroxysmal $\mathrm{AF}$, maintenance of sinus rhythm could improve QOL! ${ }^{10-12}$ Rate control of permanent $\mathrm{AF}$ is, however, not free from concerns. First, adequate control of the resting heart rate

(Received February 16, 2006; revised manuscript received April 24, 2006; accepted May 17, 2006)

The Second Department of Internal Medicine, University of Toyama, Toyama, *The Cardiovascular Institute, Tokyo, **The Division of Cardiology, Osaka General Medical Center, Osaka, ${ }^{\dagger}$ The Second Department of Internal Medicine, Fukuoka University, Fukuoka, ttThe Department of Cardiology and Internal Medicine, Kitasato University School of Medicine, Sagamihara and $¥$ The Second Department of Internal Medicine, Hirosaki University, Hirosaki, Japan

Mailing address: Takeshi Yamashita, MD, The Cardiovascular Institute, 7-3-10 Roppongi, Minato-ku, Tokyo 106-0032, Japan. E-mail: yamt-tky@umin.ac.jp
(HR) does not always imply adequate HR control during exercise! ${ }^{3,14}$ Second, digitalis can not control HR adequately during exercise ${ }^{15-17}$ Third, although $\beta$-blockers (BB) can achieve adequate HR control during exercise, exercise tolerance is rather limited in comparison with calcium antagonists (CAA) ${ }^{18}$ Fourth, estimation of health-related QOL with the Short Form-36 (SF-36) questionnaire has some limitations because it is not specific for the determination of arrhythmia-related QOL of patients with AF. Therefore, we conducted a study to determine the relationship between HR control and QOL in patients with permanent AF, and the usefulness of a newly developed questionnaire specific for estimation of AF-related QOL, the Atrial Fibrillation Quality of Life Questionnaire (AFQLQ), ${ }^{19,20}$ which is being used in an on-going prospective study (J-RHYTHM)? ${ }^{2}$

\section{Methods}

Study Population and Protocol

The Quality of Life of Atrial Fibrillation study was a multicenter, prospective study starting from February 2003. Outpatients with permanent AF who had a resting HR between 60 and 80 beats/min with digitalis for more than 6 months were selected. After giving informed consent, patients underwent treadmill test, Holter electrocardiogram (ECG) and QOL questionnaire to determine the efficacy of rate control. First, the digitalized patients were divided randomly into 2 groups (ie, BB or CAA treatment). Only 


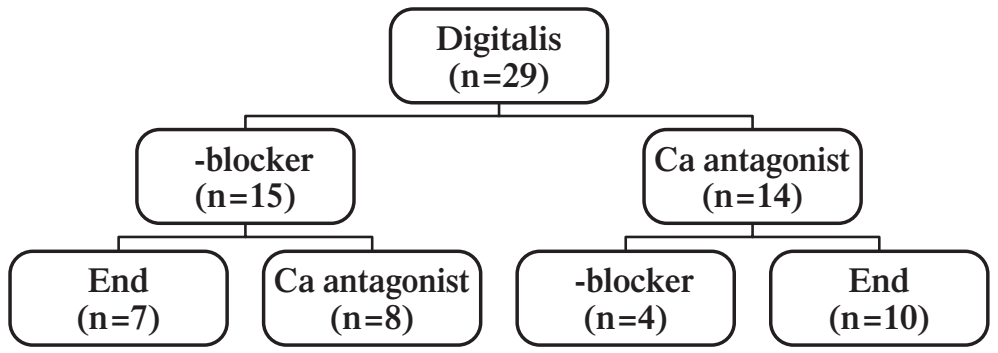

Fig 1. Protocol of the Quality of Life of Atrial Fibrillation (QOLAF) study (see text for details).

Table 1 Characteristics of the Study Groups

\begin{tabular}{lccc}
\hline \hline & $\begin{array}{c}\beta \text {-blocker } \\
\text { treatment }\end{array}$ & $\begin{array}{c}\text { Ca antagonist } \\
\text { treatment }\end{array}$ & p value \\
\hline$N$ & 19 & 22 & \\
Men & 16 & 20 & $N S$ \\
Age (years) & $68.6 \pm 8.4$ & $65.5 \pm 7.7$ & $N S$ \\
AF duration (months) & $91.8 \pm 113.0$ & $103.5 \pm 116.5$ & NS \\
Duration of therapy (days) & $79.3 \pm 34.5$ & $72.8 \pm 27.4$ & NS \\
Underlying cardiac disease & & & NS \\
$\quad$ None & 8 & 10 & \\
Valvular disease & 3 & 4 & \\
$\quad$ Cardiomyopathy & 1 & 1 & \\
$\quad$ Others & 3 & 3 & NS \\
Hypertension & 6 & 6 & NS \\
Heart failure & 2 & 3 & NS \\
(NYHA class I \& II) & & & NS \\
Comedications & 7 & 5 & \\
$\quad$ ACEI or ARB & 5 & 4 & \\
Diuretics & 15 & 4 & \\
Warfarin & 4 & & \\
Aspirin & & & \\
\hline
\end{tabular}

Data are mean $\pm S D$ or number of patients.

NS, not significant; AF, atrial fibrillation; NYHA, New York Heart Association classification; ACEI, angiotensin converting enzyme inhibitor; ARB, angiotensin receptor blocker.

verapamil was selected as the CAA, but any of bisoprolol, atenolol or metoprolol was selected as the BB according to physician's preference (Fig 1). Second, patients who consented to continue this study received the other treatment arm in a cross-over fashion. Dosage of the drugs was adjusted to achieve a resting HR at the outpatient clinic between 60 and 80 beats/min. After adequate HR control was achieved with a BB or CAA for at least 1 month, efficacy of rate control was again determined. Patients with severe underlying cardiovascular diseases other than hypertension, New York Heart Association class III or IV symptoms, and contraindication for BB were excluded.

\section{Holter ECG and Treadmill Testing}

As the Holter ECG variables, mean HR, minimum HR, maximum $H R$, and maximum and minimum RR intervals were determined automatically with a Holter analyzing system at each participating institute. As a simple index of regularization of the $\mathrm{RR}$ interval, the difference between the maximum and minimum RR intervals was determined 22 Exercise capacity was determined by symptom-limited treadmill testing with a Bruce protocol, and exercise duration and the maximum HR during exercise were determined.

\section{$Q O L$}

In the present study, 2 questionnaires were used for analyses of health-related QOL: the conventional SF-36 and
Table 2 Drugs for Heart Rate Control

\begin{tabular}{lccc}
\hline \hline & $\begin{array}{c}\beta \text {-blocker } \\
\text { treatment }\end{array}$ & $\begin{array}{c}\text { Ca antagonist } \\
\text { treatment }\end{array}$ & Cost (yen $)^{*}$ \\
\hline Digitalis & & & \\
Digoxin & $13(0.162 \mathrm{mg})$ & $15(0.167 \mathrm{mg})$ & $9.7(0.125 \mathrm{mg})$ \\
Methyldigoxin & $6(0.1 \mathrm{mg})$ & $7(0.1 \mathrm{mg})$ & $12.5(0.1 \mathrm{mg})$ \\
$\begin{array}{l}\text {-blocker } \\
\text { Bisoprolol }\end{array}$ & $13(3.75 \mathrm{mg})$ & & $176.1(5 \mathrm{mg})$ \\
Atenolol & $5(30 \mathrm{mg})$ & & $76.4(25 \mathrm{mg})$ \\
Metoprolol & $1(40 \mathrm{mg})$ & & $33.3(20 \mathrm{mg})$ \\
Calcium antagonist & & $22(120 \mathrm{mg})$ & $7.6(40 \mathrm{mg})$ \\
Verapamil & & & \\
\hline
\end{tabular}

Figs are numbers of patients, and those in parentheses are mean daily dosage of the drug.

*In 2002.

the AFQLQ ${ }^{19-21}$ SF-36 is widely used and the Japanese version $1.2^{23}$ consists of 8 subscales: (1) physical functioning $(\mathrm{PF}),(2)$ role function-physical (RP), (3) role functionemotional (RE), (4) bodily pain (BP), (5) general health perceptions $(\mathrm{GH}),(6)$ vitality $(\mathrm{VT}),(7)$ social functioning (SF), and (8) mental health (MH). These subscales were transformed to create Physical and Mental Component Summary scores (PCS and MCS, respectively), ranging from 0 to 100 points with higher scores indicating well health status, and adjusted for age and sex to have an averaged score of 50 with SD of 10, except for PCS (50.6 \pm 8.8$)$ and MCS (49.3 \pm 9.0$)$, as the Japanese national norms. ${ }^{23}$

AFQLQ was invented by the Japanese Society of Electrocardiology as a QOL questionnaire specific for AF ${ }^{19,20}$ It consists of 3 subscales: questions 1-6 reflect the variety and frequency of symptoms (0-24 points); questions 7-12, the severity of symptoms (0-18 points); and questions 13-26, limitations of daily and special activities and mental anxiety related to AF (0-56 points). Higher scores for each subscale indicate well health status as with SF-36.

\section{Statistical Analysis}

Data are presented as mean $\pm \mathrm{SD}$. In the present study, efficacy of HR control with monotherapy of BB or CAA was analyzed in comparison with the data obtained from digitalis treatment. Differences in mean values were analyzed using Student's paired and unpaired t-test as appropriate. As for the QOL scores, Wilcoxon's signed rank test was used to analyze changes. The relationship of changes between QOL scores and Holter ECG or treadmill testing was tested with a linear regression analysis. A p-value $<0.05$ was considered statistically significant.

\section{Results}

A total of 29 patients with permanent AF were enrolled between February 2003 and July 2004; 25 were men and 
Table 3 Variables of Holter ECG and Treadmill Testing

\begin{tabular}{|c|c|c|c|c|}
\hline & \multicolumn{2}{|c|}{$\beta$-blocker treatment } & \multicolumn{2}{|c|}{ Ca antagonist treatment } \\
\hline & Digitalis & $\beta$-blocker & Digitalis & Ca antagonist \\
\hline \multicolumn{5}{|l|}{ Holter ECG } \\
\hline Mean HR (beats/min) & $74 \pm 10$ & $78 \pm 12$ & $74 \pm 9$ & $83 \pm 14^{\dagger}$ \\
\hline Minimum HR (beats/min) & $43 \pm 14$ & $50 \pm 10 *$ & $44 \pm 12$ & $53 \pm 9^{\dagger}$ \\
\hline Maximum HR (beats/min) & $152 \pm 25$ & $141 \pm 24 *$ & $148 \pm 27$ & $152 \pm 31$ \\
\hline Maximum RR interval (s) & $2.9 \pm 0.8$ & $2.5 \pm 0.6^{\dagger}$ & $2.7 \pm 0.8$ & $2.3 \pm 0.5^{\dagger}$ \\
\hline Maximum RR minus minimum RR interval $(s)$ & $1.22 \pm 0.99$ & $0.83 \pm 0.28$ & $1.18 \pm 0.95$ & $0.75 \pm 0.22 *$ \\
\hline \multicolumn{5}{|l|}{ Treadmill testing } \\
\hline Exercise duration (s) & $407 \pm 126$ & $453 \pm 137 *$ & $430 \pm 131$ & $481 \pm 127^{*}$ \\
\hline Maximum HR (beats/min) & $165 \pm 24$ & $152 \pm 27 *$ & $163 \pm 30$ & $167 \pm 30$ \\
\hline
\end{tabular}

Data are mean $\pm S D$.

${ }^{\dagger} p<0.01,{ }^{*} p<0.05, * p<0.1$ s digitalis in each treatment group.

$E C G$, electrocardiogram; $H R$, heart rate.

Table 4 Scores for Short Form-36 and Atrial Fibrillation Quality of Life Questionnaire

\begin{tabular}{|c|c|c|c|c|}
\hline & \multicolumn{2}{|c|}{$\begin{array}{c}\beta \text {-blocker treatment } \\
(n=19)\end{array}$} & \multicolumn{2}{|c|}{$\begin{array}{l}\text { Ca antagonist treatment } \\
(n=22)\end{array}$} \\
\hline & Digitalis & $\beta$-blocker & Digitalis & Ca antagonist \\
\hline \multicolumn{5}{|l|}{ Short form-36 } \\
\hline Physical functioning & $52.8 \pm 8.1$ & $50.2 \pm 14.1$ & $50.6 \pm 7.9$ & $52.1 \pm 8.5 *$ \\
\hline Role function-physical & $51.0 \pm 11.4$ & $53.2 \pm 9.5$ & $48.6 \pm 12.1$ & $54.1 \pm 7.6^{+}$ \\
\hline Bodily pain & $57.5 \pm 5.7$ & $53.5 \pm 11.3$ & $55.3 \pm 7.2$ & $55.3 \pm 8.4$ \\
\hline General health perceptions & $49.8 \pm 8.1$ & $47.1 \pm 10.5$ & $47.7 \pm 6.1$ & $49.1 \pm 7.9$ \\
\hline Vitality & $50.7 \pm 8.5$ & $47.3 \pm 10.7$ & $49.6 \pm 10.0$ & $49.7 \pm 8.4$ \\
\hline Social functioning & $52.2 \pm 8.5$ & $50.0 \pm 13.5$ & $49.7 \pm 10.8$ & $52.4 \pm 9.5$ \\
\hline Role function-emotional & $52.7 \pm 7.2$ & $50.8 \pm 10.0$ & $49.7 \pm 10.2$ & $52.7 \pm 7.9 *$ \\
\hline Mental health & $52.0 \pm 7.3$ & $48.0 \pm 11.5$ & $51.6 \pm 9.1$ & $52.7 \pm 7.4$ \\
\hline Physical component summary scores & $47.7 \pm 6.1$ & $49.8 \pm 8.4$ & $47.1 \pm 5.1$ & $49.3 \pm 5.3 *$ \\
\hline Mental component summary scores & $51.2 \pm 6.3$ & $49.7 \pm 6.9$ & $51.0 \pm 7.4$ & $50.4 \pm 6.1$ \\
\hline \multicolumn{5}{|c|}{ Atrial Fibrillation Quality of Life Questionnaire } \\
\hline$Q 1-6$ & $17.4 \pm 7.2$ & $19.2 \pm 5.3$ & $15.1 \pm 6.8$ & $17.0 \pm 5.6^{+}$ \\
\hline$Q 7-12$ & $14.5 \pm 4.6$ & $15.4 \pm 3.0$ & $13.9 \pm 4.6$ & $14.7 \pm 3.3$ \\
\hline$Q 13-26$ & $45.8 \pm 8.2$ & $46.7 \pm 8.1$ & $45.8 \pm 7.8$ & $46.6 \pm 8.1$ \\
\hline
\end{tabular}

Data are mean $\pm S D$.

${ }^{\star} p<0.05, * p<0.1$ vs digitalis in each treatment group.

the mean age was $67 \pm 8$ years. All the participants, who had been digitalized already before entry, were randomized into the BB or CAA treatment group (Fig 1) and thereafter, 12 of the 29 patients agreed to continue the study with the other arm of therapy. All the data from Holter ECG, treadmill testing and QOL under BB or CAA treatment were analyzed with those under digitalis as the control value. Clinical characteristics did not differ between the 2 treatment groups (Table 1). Drugs used for HR control are summarized in Table 2. As for BB, two-thirds of the patients received bisoprolol. Only verapamil was selected as the CAA. Dosages of these drugs fell into the conventional dosages used in Japan. The daily medical cost was lower with CAA than with BB. No spontaneous conversion of AF into sinus rhythm occurred in any patient during the follow-up period.

\section{Holter ECG and Exercise Test}

Table 3 summarizes the variables of Holter ECG and treadmill testing. BB increased the minimum HR and tended to decrease the maximum HR $(p<0.1)$ without alterations in mean HR. On the other hand, CAA significantly increased the minimum HR and mean HR. Moreover, CAA tended to regularize the $R R$ interval $(\mathrm{p}=0.057)$ differently from BB. Both treatments significantly shortened the maximum RR interval as compared with digitalis treatment.

CAA prolonged exercise duration significantly $(\mathrm{p}<0.05)$ without changes in maximum HR (Table 3 ). However, BB only tended to prolong exercise duration $(\mathrm{p}<0.1)$, although maximum HR during exercise tended to decrease.

$Q O L$

For the whole group of patients maintained on digitalis, QOL scores for the SF-36 were close to the norms of healthy Japanese subjects (PF, 51.3 \pm 8.6 ; RP, 50.4 \pm 11.3 ; BP, 56.5 \pm 6.9 ; GH, 48.8 \pm 6.7 ; VT, 49.7 \pm 9.3 ; SF, 50.9 \pm 10.1 ;

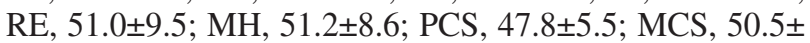
7.0). However, the greatest deviation of QOL from average was observed in PCs, which suggests that AF patients might be handicapped in performing physical activities as compared with healthy subjects. Although BB did not affect the QOL scores for SF-36, CAA improved RP significantly compared with digitalis (Table 4). As for AF-related QOL analyzed with the AFQLQ, CAA improved the variety and frequency of symptoms (Q1-6) as compared with digitalis, although $\mathrm{BB}$ did not affect it. The other 2 subscales in AFQLQ were unchanged by administration of BB or CAA (Table4).

\section{Discussion}

The present study was designed to compare the efficacy of monotherapy with either $\mathrm{BB}$ or CAA with digitalis for 
HR control and health-related QOL in patients with permanent AF. The major findings of this study are as follows. First, as compared with digitalis, BB increased the minimum HR without significant alterations in mean HR, whereas CAA increased the minimum and mean HR significantly with a trend toward regularizing the RR interval. Moreover, both treatments shortened the maximum RR interval significantly as compared with digitalis. Second, CAA prolonged exercise duration significantly compared with digitalis, although BB tended to prolong it. Third, permanent AF patients with HR controlled by digitalis had scores for the SF-36 close to the norms in Japan; ${ }^{21}$ only CAA, not BB, improved the RP of the SF-36 scores and one of the subscales of the AFQLQ ${ }^{19,20}$ as compared with digitalis.

\section{HR Control of AF}

Recent guidelines recommend a target HR between 60 and 80 beats/min at rest, and between 90 and 115 beats/min during moderate exercise ${ }^{24}$ As adequate HR at rest with digitalis does not always imply adequate HR control during exercise ${ }^{13-16}$ the addition of a BB or CAA would be required in that instance ${ }^{15-17}$ As reported previously, ${ }^{15-17}$ adequate HR control at rest does not usually imply good exercise tolerance, because $\mathrm{BB}$ and CAA prolonged exercise duration irrespective of similar HR at rest as compared with digitalis.

BB was effective in controlling HR during exercise, but rather interfered with exercise capacity, possibly because of its negative inotropic effect $14,16,18$ The present results showed that the maximum HR tended to decrease and exercise duration tended to increase with $\mathrm{BB}$ as compared with digitalis. Monotherapy with verapamil ( $40 \mathrm{mg}$ t.i.d.) was also superior to digitalis in terms of exercise tolerance, but this effect did not result from the maximum HR during exercise. These results differ from those of Farshi et $\mathrm{al}^{17}$ in some points. They recruited 12 patients with AF and tested several regimens for HR control. Although atenolol was superior to digoxin and diltiazem in controlling HR during exercise, exercise duration did not differ among the 3 regimens of monotherapy. The failure of atenolol to prolong exercise time despite a significant decrease in HR has been reported $14,16,18$ The difference between the present study and that of Farshi et al $^{17}$ deserves discussion. First, the daily dosage of atenolol in the present study was twice as high as that used in the study of Farshi et al, which might result in a remarkably decreased cardiac output, associated with lower exercise tolerance. Second was the difference in the CAA: they used diltiazem-CD once daily and we used conventional verapamil (40 mg t.i.d.).

\section{QOL of Patients With AF}

Elimination of AF by ablating the pulmonary veins can improve the QOL of patients with drug-refractory, symptomatic paroxysmal AF ${ }^{10-12}$ However, recent multicenter prospective trials with pharmacotherapy revealed that QOL determined by the SF-36 and other health-related questionnaires improved similarly with either rate control or rhythm control at the end of follow-up period, although it was impaired at baseline as compared with the norms obtained from healthy subjects ${ }^{2-5,9,25-27}$ One trial indicated that QOL actually improved at the end of follow-up when sinus rhythm was maintained pharmacologically26,27 Although the AFFIRM study did not prove a difference in the QOL between patients in sinus and AF patients? these different results could be explained by the varying baseline values in the target population.

In the present study, the QOL of patients with permanent AF was assessed with a conventional, health-related questionnaire (SF-36) and a newly developed AF-specific questionnaire (AFQLQ). The patients with permanent AF had been treated with digitalis adequately in terms of HR at entry into the study, which could possibly explain why the baseline QOL scores were close to the norms of healthy Japanese subjects. ${ }^{23}$ CAA but not BB improved the RP score of the SF-36 further in the present study. However, we should not emphasize the improvement, because the baseline values of RP in the 2 groups were somewhat different, though not significant.

The AF-specific questionnaire, AFQLQ, is newly developed to score the QOL of AF patients 19,20 It consists of 3 categories: frequency and severity of symptoms, limitations of daily and specific activities, and anxiety related to AF. AFQLQ is currently being used in an on-going prospective study (J-RHYTHM) ${ }^{21}$ On this QOL questionnaire, CAA significantly improved one of the subscales (ie, frequency and severity of symptoms (Table 4)). However, a slight difference in the baseline value, although not significant, might also affect this result, as is the case in the previous reports ${ }^{9,26}$ and our results for the SF-36. On the other hand, Johansson et al reported that verapamil regularized ventricular responses during $\mathrm{AF}$, thereby leading to a decrease in the sensation of palpitation ${ }^{28}$ In this study, we evaluated the regularization of ventricular responses simply as the difference between the maximum and minimum RR interval,2 which tended to shorten with CAA treatment $(\mathrm{p}<0.1)$ and might be related to some improvements in the SF-36 and AFQLQ scores.

Interestingly, BB did not improve the QOL scores, irrespective of the decrease in maximum HR during Holter recordings and treadmill testing. In contrast, CAA improved some QOL scores without a decrease in the maximum HR. Although these results apparently seem strange, the AFFIRM subanalysis also found that there were no significant associations between achieved HR at rest and exercise, and QOL measurements 29 However, another viewpoint should be emphasized for the management of AF patients. Another subanalysis of the AFFIRM study ${ }^{30}$ reported that overall rate control was achieved in $70 \%$ of patients given $\mathrm{BB}$ as the first drug. Moreover, during the follow-up (3.5 \pm 1.3 years), BB was required more frequently than the other 2 drugs to control HR. Accordingly, the present results for the short-term effects of drugs should be interpreted with caution.

From the point of cost-effectiveness, CAA might have some advantages over BB, as shown in Table 2. When we consider the prolongation of exercise tolerance, improvement of QOL, regularization of ventricular responses by CAA and also its total medical cost, the present results imply that CAA is superior to BB or digitalis in permanent AF patients. However, we should be careful to determine the drug for HR control in each patient, because the differences in the response to the drugs were relatively small as a group when compared with the varying responses in each subject.

\section{Study Limitations}

First, this study recruited only patients maintained on digitalis for more than 6 months. They would be stable in terms of HR control and possibly have limited symptoms 
related to AF. This could be a major selection bias possibly affecting the results. Second, only monotherapy with digitalis, BB or CAA was tested. It is well known that digitalis in combination with $\mathrm{BB}$ or CAA is highly effective in controlling HR as compared with monotherapy 16,17 The present results might have differed if combination therapy was also assessed. Third, verapamil was given 3 times a day, whereas digitalis and almost all BB are given once daily. This frequency could impair QOL. If long-acting CAA was used in the present study, it is possible that these results might differ. Fourth, women with AF usually have more impaired QOL than men, especially in the domains related to physical rather than emotional functioning? $?^{1}$ In this study, however, there were very few women. Fifth, it should be noted that the follow-up period of this study was relatively short. Therefore, the present results can not be always applied to the long-term outcome of AF patients ${ }^{30}$ Sixth, there was some difference in mean age between the BB and CAA treatment groups, although not significant, but might affect the results. Finally, the total number of patients was relatively small. More patients are required to draw a definite conclusion in terms of the QOL of patients with permanent AF.

\section{Conclusions}

The present study indicated that CAA treatment for permanent AF patients improved exercise tolerance compared with digitalis treatment. Moreover, there were improvements of some QOL scores with CAA but not with BB treatment. Therefore, from the short-term viewpoint, CAA might be preferable to digitalis when monotherapy is selected for HR control in patients with permanent AF.

\section{Acknowledgments}

The present study was supported by Chugai Pharmaceutical Co Ltd. The authors thank Katsuko Orio, Yoshihisa Oshio and Akira Yamao for their assistance. We also express our gratitude to all of the investigators who participated in this study.

\section{References}

1. Kato T, Yamashita T, Sagara K, Iinuma H, Fu LT. Progressive nature of paroxysmal atrial fibrillation: Observations from a 14-year followup study. Circ J 2004; 68: 568-572.

2. Hohnloser SH, Kuck KH, Lilienthal $\mathrm{J}$ for the PIAF Investigators. Rhythm or rate control in atrial fibrillation: Pharmacological Intervention in Atrial Fibrillation (PIAF): A randomized trial. Lancet 2000; 356: 1789-1794.

3. Van Gelder IC, Hagens VE, Bosker HA, Kingma JH, Kamp O, Kingma T, et al for the Rate Control versus Electrical cardioversion for persistent atrial fibrillation study group. A comparison of rate control and rhythm control in patients with recurrent persistent atrial fibrillation. $N$ Engl J Med 2002; 347: 1834-1840.

4. The AFFIRM Investigators. A comparison of rate control and rhythm control in patients with atrial fibrillation. $N$ Engl J Med 2002; 347: $1825-1833$

5. Carlsson J, Miketic S, Windeler J, Cuneo A, Haun S, Micus S, et al for the STAF Investigators. Randomized trial of rate-control versus rhythm-control in persistent atrial fibrillation: The Strategies of Treatment of Atrial Fibrillation (STAF) Study. J Am Coll Cardiol 2003; 41: 1690-1696.

6. Pappone C, Rosanio S, Oreto G, Tocchi M, Gugliotta F, Vicedomini $\mathrm{G}$, et al. Circumferential radiofrequency ablation of pulmonary vein ostia: A new anatomic approach for curing atrial fibrillation. Circulation 2000; 102: 2619-2628.

7. Fujiki A, Tsuneda T, Sugao M, Mizumaki K, Inoue H. Usefulness and safety of bepridil in converting persistent atrial fibrillation to sinus rhythm. Am J Cardiol 2003; 92: 472-475.

8. Fujiki A, Tsuneda T, Sakabe M, Nakagawa K, Mizumaki K, Hirai T, et al. Maintenance of sinus rhythm and recovery of atrial mechanical function after cardioversion with bepridil or in combination with aprindine in long-lasting persistent atrial fibrillation. Circ J 2004; 68: $834-839$.

9. The AFFIRM Investigators. Quality of life in atrial fibrillation: The atrial fibrillation follow-up investigation of rhythm management (AFFIRM) study. Am Heart J 2005; 149: 112-120.

10. Tada H, Naito S, Kurosaki K, Ueda M, Ito S, Shinbo G, et al. Segmental pulmonary vein isolation for paroxysmal atrial fibrillation improves quality of life and clinical outcomes. Circ J 2003; 67: 861 865 .

11. Purerfellner H, Martinek M, Aichinger J, Nesser HJ, Kempen K, Janssen JP. Quality of life restored to normal in patients with atrial fibrillation after pulmonary vein ostial isolation. Am Heart J 2004; 148: $318-325$

12. Weerasooriya R, Jais P, Hocini M, Scavee C, MacLe L, Hsu LF, et al. Effect of catheter ablation on quality of life of patients with paroxysmal atrial fibrillation. Heart Rhythm 2005; 2: 619-623.

13. Beasley R, Smith DA, McHaffie DJ. Exercise heart rates at different serum digoxin concentrations in patients with atrial fibrillation. $B M J$ 1985; 290: 9-11.

14. Bjerregaard P, Bailey WB, Robinson SE. Rate control in patients with chronic atrial fibrillation. Am J Cardiol 2004; 93: 329-332.

15. Itai T, Murayama M, Nozaki A. Comparison of effects of digoxin, propranolol or verapamil for heart rate control in patients with chronic atrial fibrillation. Jpn J Electrocardiol 1986; 6: 273-282 (in Japanese).

16. Lewis RV, McMurray J, McDevitt DG. Effects of atenolol, verapamil, and xamoterol on heart rate and exercise tolerance in digitalised patients with chronic atrial fibrillation. J Cardiovasc Pharmacol 1989; 13: $1-6$.

17. Farshi R, Kistner D, Sarma JSM, Longmate JA, Singh BN. Ventricular rate control in chronic atrial fibrillation during daily activity and programmed exercise: A crossover open-label study of five drug regimens. J Am Coll Cardiol 1999; 33: 304-310.

18. Atwood JE, Sullivan M, Forbes S, Myers J, Pewen W, Olson HG, et al. Effect of beta-adrenergic blockade on exercise performance in patients with chronic atrial fibrillation. J Am Coll Cardiol 1987; 10: 314-320.

19. Yamashita T, Kumagai K, Koretsune Y, Mitamura H, Okumura K, Ogawa $\mathrm{S}$, et al. A new method for evaluating quality of life specific to patients with atrial fibrillation: Atrial fibrillation Quality of Life Questionnaire (AFQLQ). Jpn J Electrocardiol 2003; 23: 332-343 (in Japanese).

20. Yamashita T, Komatsu T, Kumagai K, Uno K, Niwano S, Fijiki A, et al. Internal consistency and reproducibility of Atrial fibrillation Quality of Life Questionnaire (AFQLQ). Jpn J Electrocardiol 2005; 25: $488-494$ (in Japanese).

21. Yamashita T, Ogawa S, Aizawa Y, Atarashi H, Inoue H, Ohe T, et al on behalf of the J-RHYTHM Investigators. Investigation of the optimal treatment strategy for atrial fibrillation in Japan: The JRHYTHM (Japanese Rhythm Management Trial for Atrial Fibrillation) Study design. Circ J 2003; 67: 738-741.

22. Channer KS, Papouchado MA, James MA, Pitcher DW, Rees JR. Towards improved control of atrial fibrillation. Eur Heart J 1987; 8: $141-147$.

23. Fukuhara S, Suzukamo Y, Bito S, Kurokawa K. Manual of SF-36 Japanese version 1.2. Tokyo: Public Health Research Foundation, 2001.

24. Fuster V, Ryden LE, Asinger RW, Cannom DS, Crijns HJ, Frye RL, et al. ACC/AHA/ESC guidelines for the management of patients with atrial fibrillation: A report of the American College of Cardiology/American Heart Association Task Force on Practice Guidelines and the European Society of Cardiology Committee for Practice Guidelines and Policy Conferences (Committee to develop guidelines for the management of patients with atrial fibrillation) developed in collaboration with the North American Society of Pacing and Electrophysiology. Eur Heart J 2001; 22: 1852-1923.

25. Grönefeld GC, Lilienthal J, Kuck KH, Hohnloser SH for the Pharmacological Intervention in Atrial Fibrillation (PIAF) Study Investigators. Impact of rate versus rhythm control on quality of life in patients with persistent atrial fibrillation: Results from a prospective randomized study. Eur Heart J 2003; 24: 1430-1436.

26. The RACE Study Group. Effect of rate or rhythm control on quality of life in persistent atrial fibrillation. $J$ Am Coll Cardiol 2004; 43: $241-247$.

27. Hagens VE, Crijns HJGM, van Veldhuisen DJ, van den Berg MP, Rienstra M, Ranchor AV, et al for the Rate Control versus Electrical cardioversion for persistent atrial fibrillation study group. Rate control versus rhythm control for patients with persistent atrial fibrillation with mild to moderate heart failure: Results from the Rate 
Control versus Electrical cardioversion (RACE) study. Am Heart $J$ 2005; 149: $1106-1111$

28. Johansson PA, Olsson SB. Long-term oral treatment with high doses of verapamil in lone atrial fibrillation. Clin Cardiol 1984; 7: 163-170.

29. Cooper HA, Bloomfield DA, Bush DE, Katcher MS, Rawlins M, Sacco JD, et al. Relation between achieved heart rate and outcomes in patients with atrial fibrillation (from the Atrial Fibrillation Followup Investigation of Rhythm Management [AFFIRM] Study). Am J
Cardiol 2004; 93: 1247-1253.

30. Olshansky B, Rosenfeld LE, Warner AL, Solomon AJ, O'Neill G, Sharma A, et al. The Atrial Fibrillation Follow-up Investigation of Rhythm Management (AFFIRM) study: Approaches to control rate in atrial fibrillation. J Am Coll Cardiol 2004; 43: 1201-1208.

31. Paquette M, Roy D, Talajic M, Newman D, Couturier A, Yang C, et al. Role of gender and personality on quality-of life impairment in intermittent atrial fibrillation. Am J Cardiol 2000; 86: 764-768. 